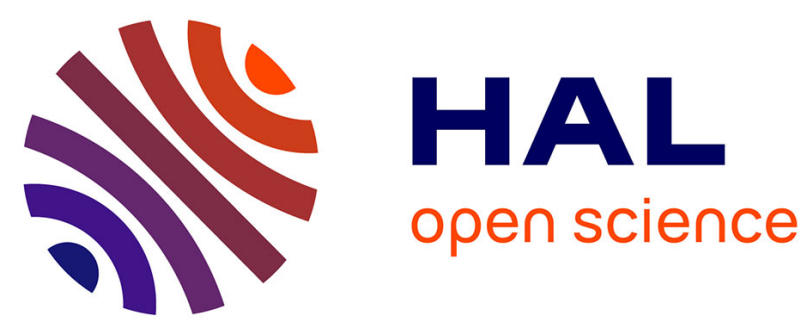

\title{
Robust power modulation for channel state information exchange
}

\author{
Chao Zhang, Vineeth Varma, Samson Lasaulce
}

\section{To cite this version:}

Chao Zhang, Vineeth Varma, Samson Lasaulce. Robust power modulation for channel state information exchange. International conference on Network Games, Control and Optimization, NETGCOOP 2016, Nov 2016, Avignon, France. hal-01407879

\section{HAL Id: hal-01407879 \\ https://hal.science/hal-01407879}

Submitted on 2 Dec 2016

HAL is a multi-disciplinary open access archive for the deposit and dissemination of scientific research documents, whether they are published or not. The documents may come from teaching and research institutions in France or abroad, or from public or private research centers.
L'archive ouverte pluridisciplinaire HAL, est destinée au dépôt et à la diffusion de documents scientifiques de niveau recherche, publiés ou non, émanant des établissements d'enseignement et de recherche français ou étrangers, des laboratoires publics ou privés. 


\title{
Robust power modulation for channel state information exchange
}

\author{
Chao ZHANG $^{1}$, Vineeth VARMA ${ }^{2}$ and Samson LASAULCE ${ }^{3}$
}

\begin{abstract}
This contribution is on the framework of a wireless network with multiple interfering transmitter-receiver pairs. We study the case where there is no direct communication channel between the multiple transmitters and the system is completely distributed (in terms of information available and decision making). Exchanging local channel state information (CSI) among the transmitters is one solution to the problem of improving the efficiency of the network. We introduce a novel power modulation scheme that facilitates reliable exchange of local CSI through the signal power strength assuming feedback of received signal strength indicator from each receiver to its transmitter. Numerical results demonstrate the value of our approach.
\end{abstract}

\section{Introduction}

This contribution is in the framework of an interference network, i.e., a wireless network with multiple transmitter-receiver pair that cause interference with each other. Typically, these transmitting and receiving devices are distributed in decision making and possibly also in terms of the information available. One of the major challenges in such networks is designing algorithms or procedures for controlling the transmit power levels of the wireless signals in order to improve the data rate (or other relevant performance metrics). The iterative water-filling algorithm (IWFA) [1][2][3] is a state-of-the art technique which relies only on locally availble information, the individual signal-to-interference plus noise ratio (SINR), and has a low computational complexity. On the other hand, one drawback of IWFA and many other similar distributed and learning algorithms (see e.g., [4][5][6]) is that convergence is not always ensured [3] and the result is typically globally inefficient.

To exploit the available feedback signal efficiently, a novel technique is given by [7]. In [7], instead of using local observations (namely, the SINR feedback realizations or received signal strength indicator (RSSI) realizations) to allow the transmitters to converge to a Nash point, one can use them to acquire global channel state information (CSI). To obtain global CSI, the key idea in [7] is to exploit the transmit power levels as information symbols and the interference as a communication mean or channel for the transmitters.

Name and Address of your Institute namedemail.address. Name and Address of your Institute name@email.address 
However, the power modulation scheme proposed in [7] results in non-negligible decoding errors. A novel technique is proposed in this paper which allows to improve the decoding efficiency, i.e., to estimate the symbol coded in the transmitted power of interfering users more precisely. Furthermore, it is interesting to note that the mapping method in [7] is only a special case of the novel technique in this paper.

The chief contributions and novelty of this work are as follows:

- We introduce the novel idea of power modulation by considering the property of the RSSI measurements and the decoding rule. This allows the transmitter to estimate the transmitted power of interfering transmitters more easily.

- If local CSI is perfectly known by each user, the conditions to completely reconstruct the transmitted power of the interfering transmitters are given.

- The proposed technique accounts for noise in the RSSI measurements (the corresponding modeling is provided in Sec. II), while IWFA-like algorithms typically assume noiseless measurements (with a few exceptions [8][3][9]), .

- We also provide numerical simulations that justify the proposed scheme.

\section{System model}

We consider a wireless network with $K \geq 2$ pairs of interfering transmitters and receivers (each pair will be referred to as a user). The technique is described for the case of two users and for a single band for ease of exposition, but an extension of this to a larger number of users or bands is possible as explained in [7].

We denote the channel gain of the link between Transmitter $i \in\{1, \ldots, K\}$ and Receiver $j \in\{1, \ldots, K\}$ by $g_{i j} \in \mathbb{R}_{\geq 0}$ (this is the fading in signal power domain and not in the amplitude domain). We use a $K \times K$ channel matrix $\mathbf{G}$ whose entries are given by the channel gains $g_{i j}$. Each channel gain, and therefore the channel matrix itself, is assumed to obey a classical block-fading variation law, i.e., the channels are assumed to be constant over a certain frame, where a frame comprises $T_{\mathrm{I}}+T_{\mathrm{II}}+T_{\mathrm{III}}$ consecutive time-slots where $T_{m} \in \mathbb{N}, m \in\{\mathrm{I}, \mathrm{II}, \mathrm{III}\}$, corresponds to the number of time-slots of Phase $m$ of the proposed procedure described in [7]. The first phase (with duration of $T_{\mathrm{I}}$ ) is reserved for estimating the local channels, i.e. each user $i$ will estimate $g_{j i}$ for all $j$. A technique to estimate this with RSSI feedback is described in [7], but the focus of this contribution is to improve the second phase, which corresponds to the time in which each user broadcasts information of his local channel gains to all the other users via his transmit power level ${ }^{1}$.

Transmitter $i, i \in\{1, \ldots, K\}$, can control its power from time-slot to time-slot and the corresponding power level at time slot $t$ is denoted by $p_{i}(t) \in\left[0, P_{\max }\right]$, with $P_{\max }$ being the maximum transmit power. $p(t)=\left(p_{1}(t), \ldots, p_{K}(t)\right)^{\mathrm{T}}$ represents the $K$-dimensional column vector formed by the transmit power lev$\overline{\mathrm{el}} \mathrm{s}, \mathrm{T}$ standing for the transpose operator.

We assume the existence of a feedback mechanism which provides each transmitter, a noisy version of the signal power received at its intended receiver for each time-slot. The signal strength observed by Receiver $i$ at time-slot $t$ is expressed as

$$
\omega_{i}(t)=g_{i i} p_{i}(t)+\sigma^{2}+\sum_{j \neq i} g_{j i} p_{j}(t)
$$

\footnotetext{
${ }^{1}$ Note that "normal" or standard communication between the transmitter and receiver of every user occurs at every phase independent of what is done in Phase I and II.
} 
where $\sigma^{2}$ is the noise variance. Receiver $i$ measures the received signal (RS) power $\omega_{i}(t)$ (for each time slot) and quantizes it using $N$ bits (the $R S$ power quantizer is denoted by $\mathscr{Q}_{\mathrm{RS}}$ ). The Receiver $i$ then sends the quantized RS power $\widehat{\omega}_{i}(t)$ as feedback to Transmitter $i$ via a noisy feedback channel. Quantization yields $\widehat{\omega}_{i}(t) \in W$, for all $i \in\{1, \ldots, K\}$, where $W=\left\{\mathrm{w}_{1}, \mathrm{w}_{2}, \ldots, \mathrm{w}_{M}\right\}$ such that $0 \leq \mathrm{w}_{1}<\mathrm{w}_{2}<\cdots<\mathrm{w}_{M}$ and $M=2^{N}$.

The feedback channel is represented by a discrete memoryless channel (DMC) whose conditional probability is represented by $\Gamma$. The distorted and noisy version ${ }^{2}$ of $\omega_{i}(t)$, which is available at Transmitter $i$, is denoted by $\widetilde{\omega}_{i}(t) \in W$; the quantity $\widetilde{\omega}_{i}(t)$ will be referred to as the received signal strength indicator $(R S S I)$. Thus, the probability that Transmitter $i$ decodes the symbol $\mathrm{w}_{\ell}$ if Receiver $i$ sent the quantized RS power $\mathrm{w}_{k}$ equals $\Gamma\left(\mathrm{w}_{\ell} \mid \mathrm{w}_{k}\right)$.

Each user $i$ has its own individual utility $u_{i}$ which is a function of the global channel matrix and all the transmit powers. Therefore, main objective of each user is to maximize its individual utility $u(p ; \mathbf{G})$ (for example, the data rate) by controlling its signal power $p_{i}$. However due to the distributed nature of the system, user $i$ does not have access to $\mathbf{G}$ and therefore can not necessarily make the optimal decision. In [7], the authors propose a novel method of exchanging local CSI resulting in each user acquiring G. We detail our contribution to this method in the following section.

\section{Exchanging local CSI via power modulation}

In this contribution, we focus on improvements to Phase II of the scheme proposed in [7], specifically with regards to power modulation. The technique in [7] is to exploit the transmit power levels as information symbols and exploit the observed interference (which is observed through the RSSI or SINR feedback) for inter-transmitter communication. The corresponding implicit communication channel is exploited to acquire global CSI knowledge namely, the matrix $\mathbf{G}$ and therefore to perform operations such as the maximization of $u(p ; \mathbf{G})$.

The process of achieving the desired power control vector is divided into three phases. In Phase I, a sequence of power levels which is known to all the transmitters is transmitted (similar to a training sequence in classical channel estimation but in the power domain), and Transmitter $i$ estimates its own channel gains (i.e., $g_{1 i}, g_{2 i}, \ldots, g_{K i}$ ) by exploiting the noisy RSSI feedback; we refer to the corresponding channel gains as local CSI. In Phase II, each transmitter informs the other transmitters about its local CSI by using power modulation. By decoding the modulated power, each transmitter can estimate the channel gains of the other users and thus, at the end of Phase II each transmitter has its own estimate of the global CSI G; the situation where transmitters have a non-homogeneous or different knowledge of global CSI is referred to as a distributed CSI scenario in [10]. In Phase III, each transmitter can then exploit global CSI to maximize (possibly in a sub-optimal manner) the network utility of interest.

The key idea of this paper is to modify the basic power modulation method to enhance the estimation quality. Firstly, we introduce the power level decoding rule and the basic power modulation method proposed in [7].

\footnotetext{
${ }^{2}$ Note that, for the sake of clarity and ease of exposition,we assume the RS power quantizer and DMC to be independent of the user index, but the proposed approach also holds in the general case.
} 


\subsection{Basic power modulation scheme}

As described before, only 2 users are active at any given time-slot in Phase II for our study. To inform the other transmitters about its knowledge of local CSI, Transmitter $i$ maps the $K$ labels of $N_{\text {II }}$ bits produced by the quantizer $Q_{i}^{\mathrm{II}}$ to a sequence of power levels $\left(p_{i}\left(T_{\mathrm{I}}+1\right), p_{i}\left(T_{\mathrm{I}}+2\right), \ldots, p_{i}\left(T_{\mathrm{I}}+T_{\mathrm{II}}\right)\right)$. As described in [7], Phase II comprises $T_{\mathrm{II}}=2$ time-slots, $K=2$ users, and that the users only exploit $L=2$ power levels during Phase II say $\mathscr{P}=\left\{P_{\min }, P_{\max }\right\}$. Further assume 1 -bit quantizers, which means that the quantizers $\mathscr{Q}_{j i}^{\mathrm{II}}$ produce binary labels. For simplicity, we assume the same quantizer $\mathscr{Q}$ is used for all the four channel gains $g_{11}, g_{12}, g_{21}$, and $g_{22}$ : if $g_{i j} \in[0, \mu]$ then the quantizer output is denoted by $g_{\min }$; if $g_{i j} \in(\mu,+\infty)$ then the quantizer output is denoted by $g_{\max }$. Therefore a simple mapping scheme for Transmitter 1 (whose objective is to inform Transmitter 2 about $\left.\left(g_{11}, g_{21}\right)\right)$ is to choose $p_{1}\left(T_{\mathrm{I}}+1\right)=P_{\min }$ if $\mathscr{Q}\left(g_{11}\right)=g_{\min }$ and $p_{1}\left(T_{\mathrm{I}}+1\right)=P_{\max }$ otherwise; and $p_{1}\left(T_{\mathrm{I}}+2\right)=P_{\min }$ if $\mathscr{Q}\left(g_{21}\right)=g_{\min }$ and $p_{1}\left(T_{\mathrm{I}}+2\right)=P_{\max }$ otherwise. Therefore, depending on the p.d.f. of $g_{i j}$, the value of $\mu$, the performance criterion under consideration, a proper mapping can chosen. For example, to minimize the energy consumed at the transmitter, using the minimum transmit power level $P_{\min }$ as much as possible is preferable; thus if $\operatorname{Pr}\left(\mathscr{Q}\left(g_{11}\right)=g_{\min }\right) \geq$ $\operatorname{Pr}\left(\mathscr{Q}\left(g_{11}\right)=g_{\max }\right)$, the power level $P_{\min }$ will be associated with the minimum quantized channel gain that is $\mathscr{Q}\left(g_{11}\right)=g_{\min }$.

At every time-slot $t \in\left\{T_{\mathrm{I}}+1, \ldots, T_{\mathrm{I}}+T_{\mathrm{II}}\right\}$, the power levels of the interfering transmitter are estimated by Transmitter $i$ as follows

$$
\underline{\widetilde{p}}_{-i}(t) \in \arg \min _{\underline{p}_{-i} \in \mathscr{P} K-1}\left|\sum_{j \neq i} p_{j} \widetilde{g}_{j i}-\left(\widetilde{\omega}_{i}(t)-p_{i}(t) \widetilde{g}_{i i}-\sigma^{2}\right)\right|
$$

where $\underline{p}_{-i}=\left(p_{1}, . ., p_{i-1}, p_{i+1}, . ., p_{K}\right)$. As for every $j, \widetilde{g}_{j i}$ is known at Transmitter $i$, the above minimization operation can be performed. When the number of users is higher, each transmitter needs to estimate $K-1$ power levels with only one observation equation, which typically induces a non-negligible degradation in terms of symbol error rate. In this situation, Phase II can be performed by scheduling the activity of all the users, such that only 2 users are active at any given time-slot in Phase II. Once all pairs of users have exchanged information on their channel states, Phase II is concluded.

\subsection{An adaptive modulation scheme with local CSI perfectly known}

Without loss of generality, we consider the case with $\mathrm{K}=2$. For consistency, we assume 1-bit quantizer of $g_{j i}$, which means that the quantizer $\mathscr{Q}_{j i}^{\mathrm{II}}$ produces binary labels. For transmitter $i$, the objective is to estimate the power emitted by another transmitter $i^{\prime}$ with $i^{\prime} \neq i$. With the proposed decoding method in the previous section, it can be seen that the decoding error is mainly brought by the consequence that with different power levels emitted, the same quantized symbols (RSSI feedback) will be achieved under some extreme conditions, such as $g_{j i}$ is very close to 0 . To avoid the occurrence that the RSSI feedbacks lie in the same quantization intervals with different transmitted power, we propose a novel power modulation scheme here. For simplicity, we analyze the received signal of receiver 1 to introduce our novel technique. Assume Phase II comprises $T_{\mathrm{II}}=2$ time-slots, where $p_{1}\left(T_{\mathrm{I}}+1\right)$ depends on $g_{11}$ and $p_{1}\left(T_{\mathrm{I}}+2\right)$ depends on $g_{21}$. The basic idea here, by denoting $j \in\{1,2\}$, is to transmit with power $p_{1}\left(T_{\mathrm{I}}+j\right)$ which is larger than $P_{\max } / 2$ if $\mathscr{Q}\left(g_{j 1}\right)=g_{\max }$, otherwise the power emitted by transmitter $1 p_{1}\left(T_{\mathrm{I}}+j\right)$ should be less than $P_{\max } / 2$, . The most important point is to choose the power $p_{1}\left(T_{\mathrm{I}}+j\right)$ such that $\omega_{1}\left(T_{\mathrm{I}}+j\right)=g_{11} p_{1}\left(T_{\mathrm{I}}+j\right)+g_{21} p_{2}\left(T_{\mathrm{I}}+\right.$ 
$j)+\sigma^{2}$ lie in different quantization intervals with $p_{2}\left(T_{\mathrm{I}}+j\right) \geq P_{\max } / 2$ and $p_{2}\left(T_{\mathrm{I}}+j\right)<P_{\max } / 2$. Intuitively, it can be easily found that if $g_{11} p_{1}\left(T_{\mathrm{I}}+j\right)+\frac{1}{2} \times g_{21} P_{\max }+\sigma^{2}$ exactly equals to a quantization interval bound, the point mentioned above can be perfectly fulfilled. Denoting the quantization interval bounds of RSSI quantizer by $\left\{t_{1}, t_{2}, \ldots, t_{2^{M}+1}\right\}$ (where $\omega \in\left[t_{m}, t_{m+1}\right)$ is quantized as $w_{m}$ ), the power $p_{1}\left(T_{\mathrm{I}}+j\right)$ can be selected as follows:

$$
p_{1}\left(T_{\mathrm{I}}+j\right)= \begin{cases}\min \left(\frac{1}{2} P_{\max }+\frac{1}{g_{11}}\left(t_{a}-\frac{1}{2} g_{21} P_{\max }-\frac{1}{2} g_{11} P_{\max }-\sigma^{2}\right), P_{\max }\right) & \text { if } \mathscr{Q}\left(g_{j 1}\right)=g_{\max } \\ \min \left(\frac{1}{g_{11}}\left(t_{b}-\frac{1}{2} g_{21} P_{\max }-\sigma^{2}\right), \frac{1}{2} P_{\max }\right) & \text { else }\end{cases}
$$

where the index $a$ fulfills $t_{a-1} \leq \frac{1}{2} g_{21} P_{\max }+\frac{1}{2} g_{11} P_{\max }+\sigma^{2}<t_{a}$ and the index $b$ fulfills $t_{b-1} \leq \frac{1}{2} g_{21} P_{\max }+$ $\sigma^{2}<t_{b}$.

Proposition 1. Assume the local CSI is perfectly known by each user and the effect of the largest quantization interval is ignored, if the two conditions below are always satisfied:

$$
\text { (i) } \frac{1}{2} P_{\max }+\frac{1}{g_{11}}\left(t_{a}-\frac{1}{2} g_{21} P_{\max }-\frac{1}{2} g_{11} P_{\max }-\sigma^{2}\right) \leq P_{\max }
$$

$$
\frac{1}{g_{11}}\left(t_{b}-\frac{1}{2} g_{21} P_{\max }-\sigma^{2}\right)<\frac{1}{2} P_{\max }
$$

then transmitter 1 can always reconstruct the power emitted by transmitter 2 without error, i.e. $p_{2}$ can be decoded perfectly by transmitter 1 .

Proof. If $g_{11} p_{1}\left(T_{\mathrm{I}}+j\right)+g_{21} \frac{P_{\max }}{2}+\sigma^{2}$ can always achieve the boundary of the quantization interval, the $p_{2}$ can be perfectly decoded since 2 possible values of $p_{2}$ are belongs to $\left(0, \frac{P_{\max }}{2}\right)$ and $\left(\frac{P_{\max }}{2}, P_{\max }\right)$ respectively. The first condition above indicates that there exists $p_{1}\left(T_{\mathrm{I}}+j\right) \in\left(\frac{P_{\max }}{2}, P_{\max }\right)$ such that $g_{11} p_{1}\left(T_{\mathrm{I}}+j\right)+$ $g_{21} \frac{P_{\max }}{2}+\sigma^{2}$ equals to a boundary of the quantization interval, and the second condition above indicates that there exists $p_{1}\left(T_{\mathrm{I}}+j\right) \in\left(0, \frac{P_{\max }}{2}\right)$ such that $g_{11} p_{1}\left(T_{\mathrm{I}}+j\right)+g_{21} \frac{P_{\max }}{2}+\sigma^{2}$ equals to a boundary of the quantization interval.

Considering the 2 conditions above, it can be observed that the conditions are difficult to be fulfilled if the channel gain $g_{11}$ is too small. Even it is known that $g_{11}$ follows the exponential distribution in our scenario, it is reasonable to set a minimum value $g_{\min }$ for the channel gain since it is usually met in practical scenario.

Proposition 2. Assume the local CSI is perfectly known by each user and the effect of the largest quantization interval is ignored, if each realization of the channel gain $g_{11}$ is larger than $g_{\min }=\frac{2 \Delta}{P_{\max }}$ where the longest length of the quantization interval (except the interval $\left(t_{2^{N}}, t_{2^{N}+1}\right)$ ) is denoted as $\Delta=\max _{i \in\left\{1, \ldots, 2^{N}-1\right\}}\left(t_{i+1}-t_{i}\right)$, then transmitter 1 can always reconstruct the power emitted by transmitter 2 without error. Specially, if the $R S S I$ is uniform quantized with step $d$, then the minimum value can be expressed as $g_{\min }=\frac{2 d}{P_{\max }}$.

Proof. Omitted. 


\subsection{An adapted modulation scheme with noisy local CSI}

However, the scheme detailed in the previous section is not robust to noisy local CSI. Since the local CSI can not be perfectly acquired ( $g_{i j}$ is not perfectly known to the transmitters), it would be difficult to calculate the $p_{1}\left(T_{\mathrm{I}}+j\right)$ such that

$$
g_{11} p_{1}\left(T_{\mathrm{I}}+j\right)+\frac{1}{2} g_{21} P_{\max }+\sigma^{2}=t_{a^{\prime}}
$$

where $a^{\prime} \in\left\{2, \ldots, 2^{N}\right\}$. Using the same rule in Sec 3.2 , with noisy (estimated) $\widetilde{g}_{11}$ and $\widetilde{g}_{21}$, the $p_{1}\left(T_{\mathrm{I}}+j\right)$ can be only obtained by:

$$
\widetilde{g}_{11} p_{1}\left(T_{\mathrm{I}}+j\right)+\frac{1}{2} \widetilde{g}_{21} P_{\max }+\sigma^{2}=t_{a^{\prime}}
$$

Therefore, it can not be guaranteed that the real RSSI when the other transmiiter uses $P_{\max } / 2$ exactly equals to the interval bound due to the noise in the local CSI. To compromise for this, we propose a heuristic technique to improve the decoding rate by combining the schemes used in Sections 3.1 (which is robust to the noise in local CSI estimation but has lower decoding success rate even with perfect local CSI) and Section 3.2 (which has a very high decoding success rate with perfect local CSI, but is not robust to noise). We are able to achieve this by introducing an "offset" $x$, which we will describe in this section.

Define $\widetilde{g}_{11}=g_{11}+z_{11}$ and $\widetilde{g}_{21}=g_{21}+z_{21}$ (the noisy estimate of local CSI available at the transmitter), (7) can be rewritten as:

$$
g_{11} p_{1}\left(T_{\mathrm{I}}+j\right)+\frac{1}{2} g_{21} P_{\max }+\sigma^{2}+\left(z_{11} p_{1}\left(T_{\mathrm{I}}+j\right)+\frac{1}{2} z_{12} P_{\max }\right)=t_{a^{\prime}}
$$

Nevertheless, no matter what is the distribution of the noise, it can be found that with a probability $q$ that

$$
\operatorname{Pr}\left(\left|z_{11} p_{1}\left(T_{\mathrm{I}}+j\right)+\frac{1}{2} z_{12} P_{\max }\right| \geq c\right) \leq q
$$

where $c$ is a constant related to the probability $q$ and the noisy local CSI. Combine (8)(9), it can be concluded $\operatorname{Pr}(A) \geq q$ where the event $\mathrm{A}$ is defined as

$$
t_{a^{\prime}}-c \leq g_{11} p_{1}\left(T_{\mathrm{I}}+j\right)+\frac{1}{2} g_{21} P_{\max }+\sigma^{2} \leq t_{a^{\prime}}+c
$$

Equivalently, the (10) can be rewritten as:

$$
\begin{aligned}
& g_{11} p_{1}\left(\left(T_{\mathrm{I}}+j\right)+g_{21}\left(\frac{1}{2} P_{\max }+\frac{c}{g_{21}}\right)+\sigma^{2} \geq t_{a}^{\prime}\right. \\
& g_{11} p_{1}\left(\left(T_{\mathrm{I}}+j\right)+g_{21}\left(\frac{1}{2} P \max -\frac{c}{g_{21}}\right)+\sigma^{2} \leq t_{a}^{\prime}\right.
\end{aligned}
$$

(11) and (12) imply that even though the noisy local CSI reduce the accuracy of the emitted power, the received signal $\omega_{1}\left(T_{\mathrm{I}}+j\right)=g_{11} p_{1}\left(T_{\mathrm{I}}+j\right)+g_{21} p_{2}\left(T_{\mathrm{I}}+j\right)+\sigma^{2}$ lie in different quantization intervals with probability $q^{\prime} \geq q$ for $p_{2}\left(T_{\mathrm{I}}+j\right) \geq\left(\frac{1}{2} P \max +\frac{c}{g_{21}}\right)$ and $p_{2}\left(T_{\mathrm{I}}+j\right) \leq\left(\frac{1}{2} P \max -\frac{c}{g_{21}}\right)$. To improve the robustness of the transmission, the offset $x(x \leq 0.5)$ will be implemented such that transmitting with power $p_{1}\left(T_{\mathrm{I}}+j\right)$ which is larger than $\left(\frac{1}{2}+x\right) P_{\max }$ if $\mathscr{Q}\left(g_{j 1}\right)=g_{\max }$, transmitting with power $p_{1}\left(T_{\mathrm{I}}+j\right)$ which is less than $\left(\frac{1}{2}-x\right) P_{\max }$ if $\mathscr{Q}\left(g_{j 1}\right)=g_{\min }$. Induced by noisy local CSI, the power $p_{1}\left(T_{\mathrm{I}}+j\right)$ can be redetermined 
as follows:

$$
p_{1}\left(T_{\mathrm{I}}+j\right)= \begin{cases}\min \left(\left(\frac{1}{2}+x\right) P_{\max }+\frac{1}{\widetilde{g}_{11}}\left(t_{a}^{*}-\frac{1}{2} \widetilde{g}_{21} P_{\max }-\left(\frac{1}{2}+x\right) g_{11} P_{\max }-\sigma^{2}\right), P_{\max }\right) & \text { if } \mathscr{Q}\left(\widetilde{g}_{j 1}\right)=g_{\max } \\ \min \left(\frac{1}{\widetilde{g}_{11}}\left(t_{b}^{*}-\frac{1}{2} \widetilde{g}_{21} P_{\max }-\sigma^{2}\right),\left(\frac{1}{2}-x\right) P_{\max }\right) & \text { else }\end{cases}
$$

where the index $a^{*}$ fulfills $t_{a^{*}-1}<\frac{1}{2} \widetilde{g}_{21} P_{\max }+\left(\frac{1}{2}+x\right) \widetilde{g}_{11} P \max +\sigma^{2} \leq t_{a}^{*}$ and the index $b^{*}$ fulfills $t_{b^{*}-1}<$ $\frac{1}{2} \widetilde{g}_{21} P_{\max }+\sigma^{2} \leq t_{b}^{*}$. Transmitting with 0 and $P_{\max }$ is a special case of the new scheme corresponding to $x=0.5$. The optimal $x$ can be calculated by performing numerical simulations.

Remark 1. In the Section 3.2 and 3.3, it is assumed that the error of DMC is negligible, i.e. $\widehat{\omega}_{i}=\widetilde{\omega}_{i}$. The approach to reduce the influence of the error induced by DMC will be studied in future works.

Remark 2. (extension to the multi-band scenario). As explained in the beginning of this paper, each band performs in parallel like the single-band case. Since there are power constraints for each transmitter, the modulated power should satisfy $\sum_{s=1}^{S} p_{i}^{s}(t) \leq P_{\max }$.

\section{Numerical analysis}

In this section, as a first step, we start with providing simulations which result from the adapted power modulation when local CSI is perfectly known. To make a coherent comparison with the simple power modulation in [7], the quality of decoding will be evaluated by considering the distortion and sum-rate utility. As a second step, we study the scenario with imperfect local CSI and analyze the influence of the offset, which is introduced to assure the robustness of the system.

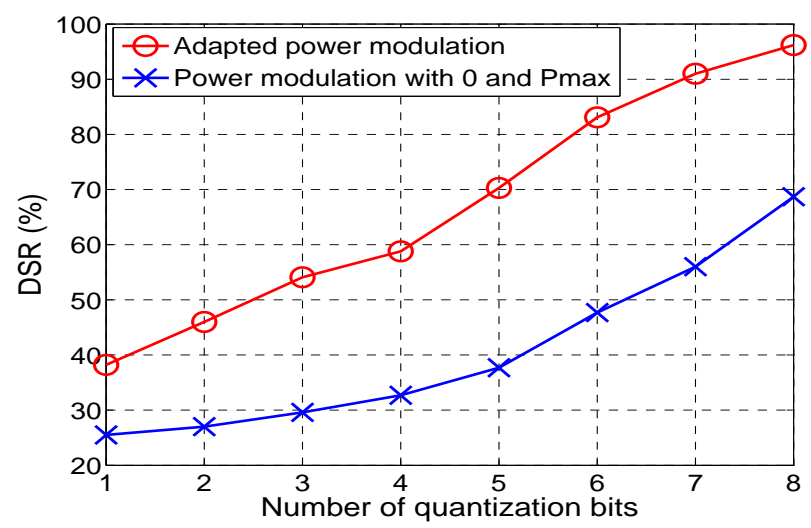

Fig. $1 \mathrm{~K}=2, \mathrm{~S}=1, \mathrm{SNR}=30 \mathrm{~dB}, \varepsilon=0$, perfect local CSI, 1 quantization bit for channel gain, we observe that the adapted modulation scheme brings higher DSR in low and high resolution case. 


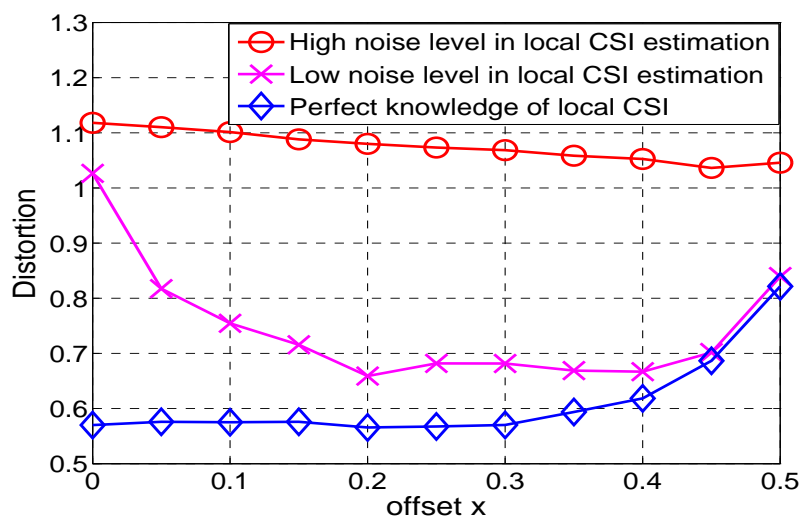

Fig. $2 \mathrm{~K}=2, \mathrm{~S}=1, \mathrm{SNR}=30 \mathrm{~dB}, \varepsilon=0$, noisy local CSI, 8 quantization bits for RSSI, 1 quantization bit for channel gain, different noise level have different optimum offset values.

Firstly, we focus on the scenario with perfect CSI. The novel modulation scheme is designed to reduce the error probability of decode. To estimate the decoding quality, the decoding success rate (DSR) is introduced, which represent the probability that the transmitter $i$ correctly reconstruct the transmitted power by all the other transmitters in all time-slots. For the sake of simplicity, we consider the $\mathrm{K}=2, \mathrm{~S}=1$ scenario. The channel gain of direct channel $g_{i i}$ follows the exponential distribution with expectation 1, where the channel gain of cross channel $g_{j i}(j \neq i)$ follows the exponential distribution with expectation 0.1, i.e. $f\left(g_{i i}\right)=$ $\exp \left(-g_{i i}\right)$ and $f\left(g_{j i}\right)=10 \exp \left(-10 g_{j i}\right)$. Furthermore, each channel is assumed to be independent. We use the uniform quantizer for the RSSI feedback and Maximum entropy quantizer (see [7]) for the channel gain in this part. In Fig. 1, it can be seen that the adapted power modulation achieves a much better performance in terms of DSR with both low and high quantization bits. Moreover, with the adapted modulation scheme, Fig. 1 shows that the DSR increases when we have more quantization bits, and it tends to 1 when the number of quantization bit is large.

Secondly, we take into account the noisy local CSI scenario. As described in [7], the local CSI can be estimated by RSSI feedback with training matrix. To distinguish the noise level here, we define the high noise level when we use 4 quantization bits of RSSI estimating the local CSI and the low noise level when we use 8 quantization bits of RSSI estimating the local CSI. Fig. 2 illustrate the offset is useful to reduce the estimation distortion, which is defined by

$$
\text { Distortion }=\mathbb{E}\left[\left\|\mathbf{G}-\widetilde{\mathbf{G}}_{i}\right\|^{2}\right]
$$

Also, it can be observed that different noise level has different optimum offset value. When noise level is low, the optimum offset value is between 0.2 and 0.3 , where 0 and $P_{\max }$ becomes a good solution when noise level is high since less choices can avoid the decoding error. Meanwhile, as the objective of the adapted modulation scheme in perfect local CSI scenario, the red curve illustrates our scheme (offset 0 ) beats the simple modulation scheme (offset 0.5 ). 


\section{Conclusion}

In this contribution, we have proposed a novel power modulation scheme that improves the inter-transmitter communication efficiency of the technique introduced in [7]. Under the assumption of noiseless feedback of the quantized received signal strength, our simulations show that a significant improvement in the distortion or decoding success rate can be achieved both for the case where perfect local CSI and noisy local CSI is available. A relevant extension to this scheme would be to account for the noise in feedback (in the DMC).

\section{References}

1. Wei Yu, George Ginis, and John M Cioffi. Distributed multiuser power control for digital subscriber lines. Selected Areas in Communications, IEEE Journal on, 20(5):1105-1115, 2002.

2. G. Scutari, D.P. Palomar, and S. Barbarossa. The MIMO iterative waterfilling algorithm. IEEE Trans. Signal Process., 57(5):1917-1935, May 2009.

3. Panayotis Mertikopoulos, Elena V Belmega, Aris L Moustakas, and Samson Lasaulce. Distributed learning policies for power allocation in multiple access channels. Selected Areas in Communications, IEEE Journal on, 30(1):96-106, 2012.

4. L. Rose, S. Lasaulce, S.M. Perlaza, and M. Debbah. Learning equilibria with partial information in decentralized wireless networks. IEEE Commun. Mag., 49(8):136-142, August 2011.

5. S. Lasaulce and H. Tembine. Game Theory and Learning for Wireless Networks: Fundamentals and Applications. Academic Press, Waltham, MA, 2011.

6. Giacomo Bacci, Samson Lasaulce, Walid Saad, and Luca Sanguinetti. Game theory for networks: A tutorial on gametheoretic tools for emerging signal processing applications. IEEE Signal Process. Mag., 33(1):94-119, 2016.

7. Vineeth S. Varma, Samson Lasaulce, and Chao Zhang. Power modulation: Application to inter-cell interference coordination. In EUSIPCO. IEEE, 2015.

8. Amod J. G. Anandkumar, Animashree Anandkumar, Sangarapillai Lambotharan, and Jonathon A. Chambers. Robust rate maximization game under bounded channel uncertainty. IEEE Transactions on Vehicular Technology, 60(9):4471-4486, 2011.

9. Pierre Coucheney, Bruno Gaujal, and Panayotis Mertikopoulos. Distributed optimization in multi-user MIMO systems with imperfect and delayed information. In ISIT '14: Proceedings of the 2014 IEEE International Symposium on Information Theory, 2014.

10. Paul De Kerret and David Gesbert. Degrees of freedom of the network mimo channel with distributed csi. Information Theory, IEEE Transactions on, 58(11):6806-6824, 2012. 